\title{
Oncogenes, G-Proteins
}

National Cancer Institute

\section{Source}

National Cancer Institute. Oncogenes, G-Proteins. NCI Thesaurus. Code C18340.

Mutated forms of G-protein genes which encode a diverse group of regulatory proteins

that act as molecular switches. Under normal cellular conditions, the activity of these proteins is tightly controlled. Activation of G-protein proto-oncogenes via mutation results in G-protein oncogenes. These aberrant genes express protein products that disrupt normal cell function. 